\title{
Langmuir
}

pubs.acs.org/Langmuir

(C) 2009 American Chemical Society

\section{Evidence for Potassium Carbonate Crystallites on Air-Cleaved Mica Surfaces}

\author{
Frank Ostendorf, ${ }^{\dagger}$ Carsten Schmitz, ${ }^{\dagger}$ Sabine Hirth,${ }^{\dagger}$ Angelika Kühnle, ${ }^{*}{ }^{\dagger}$ Jacek J. Kolodziej, ${ }^{\dagger}$ and \\ Michael Reichling ${ }^{\dagger}$ \\ ${ }^{\dagger}$ Fachbereich Physik, Universität Osnabrück, Barbarastr. 7, 49076 Osnabrück, Germany, and Research Centre \\ for Nanometer-Scale Science and Advanced Materials (NANOSAM), Faculty of Physics, Astronomy and \\ Computer Science, Jagiellonian University, ul. Reymonta 4, 30-059 Krakow, Poland
}

\section{Received April 14, 2009}

\begin{abstract}
Air-cleaved mica surfaces exhibit a high density of nanometer or micrometer size particles that have been ascribed to potassium carbonate formed as a reaction product of carbonaceous gases with potassium ions. Unambiguous evidence for this assignment has, however, never been presented. We study air-cleaved mica surfaces by high-resolution noncontact atomic force microscopy (NC-AFM) in ultrahigh vacuum to reveal the detailed structure of such precipitates on the surface. Among a large number of irregularly shaped surface structures, we find flat, hexagonally shaped islands exhibiting two different patterns on their surfaces, namely a rectangular atomic corrugation pattern and a hexagonal moiré structure. The unit cell of the rectangular pattern corresponds to the dimensions of the potassium carbonate bulk structure and is found on high crystallites. The moiré structure solely appears on very flat islands and is caused by the interference of the potassium carbonate lattice periodicity and the lattice periodicity of the underlying mica substrate. Both results strongly point to the presence of potassium carbonate crystallites on air-cleaved mica surfaces.
\end{abstract}

\section{Introduction}

Muscovite mica is one of the most investigated minerals serving as a prototype material in many areas of fundamental and applied research. The mica surface is a well-established substrate for (macro) molecule adsorption ${ }^{1-6}$ as well as a model system for studying reactions of water on insulating substrates. ${ }^{7-11}$ Knowledge on the interaction of water with Muscovite mica is important for describing processes of dissolution and crystal growth, precipitation, and biomineralization and helps understanding geochemical $^{12}$ and biological processes. ${ }^{13}$ Furthermore, this research has impact on mica-related technologies like cloud seeding $^{14,15}$ and petroleum catalysis. ${ }^{16,17}$

Muscovite mica is a rock-forming mineral and belongs to the family of clay minerals. Mica is famous for providing a molecularly flat surface due to its perfect cleavage parallel to

*Corresponding author: E-mail: kuehnle@uos.de. Phone: + 49541 969-2642. Fax: + $49541969-12642$.

(1) Pfau, A.; Schrepp, W.; Horn, D. Langmuir 1999, 15, 3219.

(2) Xia, C.; Fan, X.; Locklin, J.; Advincula, R. C.; Gies, A.; Nonidez, W. J. Am. Chem. Soc. 2004, 126, 8735 .

(3) Diez-Perez, I.; Luna, M.; Teheran, F.; Ogletree, D. F.; Sanz, F.; Salmeron, M. Langmuir 2004, 20, 1284.

(4) Chen, J.; Ratera, I.; Ogletree, D. F.; Salmeron, M.; Murphy, A. R.; Frechet,

J. M. J. Langmuir 2005, 21, 1080 .

(5) Gunari, N.; Schmidt, M.; Janshoff, A. Macromolecules 2006, 39, 2219.

(6) Kumaki, J.; Hashimoto, T. J. Am. Chem. Soc. 2003, 125, 4907.

(7) Hu, J.; Xiao, X. D.; Ogletree, D. F.; Salmeron, M. Science 1995, 268, 267.

(8) Odelius, M.; Bernasconi, M.; Parrinello, M. Phys. Rev. Lett. 1997, 78, 2855.

(9) Park, S. H.; Sposito, G. Phys. Rev. Lett. 2002, 89, 085501.

(10) Park, C.; Fenter, P. A.; Nagy, K. L.; Sturchio, N. C. Phys. Rev. Lett. 2006, 97, 016101 .

(11) Verdaguer, A.; Sacha, G. M.; Bluhm, H.; Salmeron, M. Chem. Rev. 2006, 106,1478 .

(12) Sposito, G.; Skipper, N. T.; Sutton, R.; Park, S. H.; Soper, A. K.; Greathouse, J. A. Proc. Natl. Acad. Sci. U.S.A. 1999, 96, 3358.

(13) Israelachvili, J. Intermolecular \& Surface Forces; 2nd ed., reprinted ed.; Academic Press: London, 1991; Vol. XXI.

(14) Caslavsk, J.; Vedam, K. J. Appl. Phys. 1971, 42, 516.

(15) Edwards, G. R.; Evans, L. F.; Zipper, A. F. Trans. Faraday Soc. 1970, 66, 220.

(16) Karaborni, S.; Smit, B.; Heidug, W.; Urai, J.; vanOort, E. Science 1996, 271,1102 .

(17) Boek, E. S.; Coveney, P. V.; Skipper, N. T. J. Am. Chem. Soc. 1995, 117, 12608. the $\{001\}$-plane. Mica crystallizes in a monoclinic, layered crystal structure and is commonly associated with the chemical formula $\mathrm{KAl}_{2}\left(\mathrm{Si}_{3} \mathrm{Al}\right) \mathrm{O}_{10}(\mathrm{OH})_{2}$. The bulk crystal structure is formed by aluminosilicate layers permanently negatively charged due to a substitution of one-fourth of $\mathrm{Si}^{4+}$ ions by $\mathrm{Al}^{3+}$ ions. The charge is compensated by electrostatically bound interlayer $\mathrm{K}^{+}$ions. These potassium ions are weakly bound, resulting in the easy cleavage of the crystal between two aluminosilicate layers. It is commonly believed that upon cleavage the aluminosilicate layers remain intact, whereas the potassium ions are statistically shared between the two freshly cleaved mica sheets, giving rise to an electrostatically charged surface. ${ }^{18}$

When cleaved in air, the surface is known to exhibit a high density of precipitates that have been interpreted as reaction products of carbonaceous gases reacting with the surface potassium ions. ${ }^{19}$ Upon exposure of the mica surface to humid air and subsequent drying for 2 weeks, the formation of larger crystallite structures has been observed by electron microscopy. ${ }^{20}$ These structures have been associated with crystal growth of potassium carbonate from water, potassium ions, and carbon dioxide. ${ }^{21}$

In the present study, we unveil atomic-scale details of regularly shaped crystallites observed on air-cleaved mica surfaces by noncontact atomic force microscopy (NC-AFM) imaging. Our experiments reveal unit cell dimensions of the crystallite structure perfectly in agreement with the crystal structure of potassium carbonate. Moreover, a moiré pattern is observed that is formed by the superposition of the potassium carbonate lattice periodicity and the lattice periodicity of the underlying mica substrate, further corroborating the conclusion of potassium carbonate growth.

(18) Müller, K.; Chang, C. C. Surf. Sci. 1969, 14, 39.

(19) Ostendorf, F; Schmitz, C.; Hirth, S.; Kühnle, A.; Kolodziej, J. J.; Reichling, M. Nanotechnology 2008, 19, 305705.

(20) Christenson, H. K.; Israelachvili, J. N. J. Colloid Interface Sci. 1987, 117, 576.

(21) Israelachvili, J. N.; Alcantar, N. A.; Maeda, N.; Mates, T. E.; Ruths, M. Langmuir 2004, 20, 3616. 


\section{Experimental Methods}

Experiments are performed in an ultrahigh-vacuum (UHV) system with a base pressure below $10^{-10}$ mbar consisting of a preparation chamber and a measurement chamber equipped with a commercial NC-AFM instrument (UHV 750 from RHK, Troy, $\mathrm{MI}$ that is operated in the noncontact mode as described in our earlier studies. ${ }^{22}$ As force sensors, we use p-doped silicon cantilevers (PPP-QFMR from Nanosensors, Neuchâtel, Switzerland) with a resonance frequency of about $70 \mathrm{kHz}$, a spring constant of about $2.8 \mathrm{~N} / \mathrm{m}$, and a $Q$ value above 80000 in UHV. The cantilever oscillation amplitude is kept constant at a level of $10-15 \mathrm{~nm}$. All tips are covered by a native oxide layer when purchased. We use these tips without any treatment except a bakeout at $400 \mathrm{~K}$ in UHV to remove volatile contaminants prior to AFM imaging. When approaching the surface with the oscillating cantilever, the tip-surface interaction shifts the actual resonance frequency, $f_{\text {res }}$, relative to the eigenfrequency, $f_{\text {eigen }}$, of the cantilever, commonly referred to as detuning, $\Delta f=$ $f_{\text {res }}-f_{\text {eigen }}$. In our experiments, we use two modes of operation, namely the constant height mode and the constant detuning mode often referred to as topography mode. ${ }^{23}$ It turned out that only the constant height imaging mode is capable of providing highest resolution images revealing nanoscopic details that we observe on the mica surface. This mode can, however, only be used when scanning on fairly flat terraces. Images recorded in the constant height mode directly provide the detuning signal $\Delta f$ but do not provide any topographic information. For imaging high clusters and crystallites, the constant detuning mode is used where the detuning signal is kept constant by adjusting the tip-surface distance, and the error signal of the regulation loop provides the topography signal $z$.

All images presented in this study were recorded at room temperature. Characteristic lateral drift velocities during imaging were in the range of 3-7 pm/s. For best imaging contrast on air-cleaved surfaces, the tip-surface electrostatic interaction is minimized by a Kelvin probe compensation with a variable sample bias voltage of typically -2.5 to $+2.5 \mathrm{~V}$ applied between the metallic sample support and the chamber ground connected to the tip. ${ }^{24}$

We use mica samples of best available quality (Hi grade Quality) purchased from Plano GmbH (Wetzlar, Germany). The mica sheets are cut and cleaved as detailed in a previous publication. ${ }^{19}$

Prior to NC-AFM experiments, air-cleaved mica samples are degassed at temperatures ranging from 500 to $560 \mathrm{~K}$ following recipes reported in the literature. ${ }^{19,25-29}$ The results presented here originate from more than 15 independent measurements, each starting with a freshly cleaved mica sample. Several of these measurements were used for finding the right preparation conditions, i.e., without achieving high-resolution results. ${ }^{19}$ However, after identifying the right preparation recipe, six independent mica samples revealed high-resolution results as shown here.

\section{Results and Discussion}

Air-cleaved mica surfaces always exhibit a high density of small particles. ${ }^{19,21}$ The density of these small particles amounts to several ten thousands per $\mu \mathrm{m}^{2}$. Depending on the scan frame size,

(22) Hirth, S.; Ostendorf, F.; Reichling, M. Nanotechnology 2006, 17, S148.

(23) Gritschneder, S.; Namai, Y.; Iwasawa, Y.; Reichling, M. Nanotechnology 2005, 16, S41.

(24) Gritschneder, S.; Reichling, M. Nanotechnology 2007, 18, 044024.

(25) Ferrero, S.; Piednoir, A.; Henry, C. R. Nano Lett. 2001, 1, 227.

(26) Derose, J. A.; Thundat, T.; Nagahara, L. A.; Lindsay, S. M. Surf. Sci. 1991, 256, 102.

(27) Proehl, H.; Nitsche, R.; Dienel, T.; Leo, K.; Fritz, T. Phys. Rev. B 2005, 71, 165207.

(28) Proehl, H.; Dienel, T.; Nitsche, R.; Fritz, T. Phys. Rev. Lett. 2004, 93, 097403.

(29) Lee, S. M.; Krim, J. Thin Solid Films 2005, 489, 325.
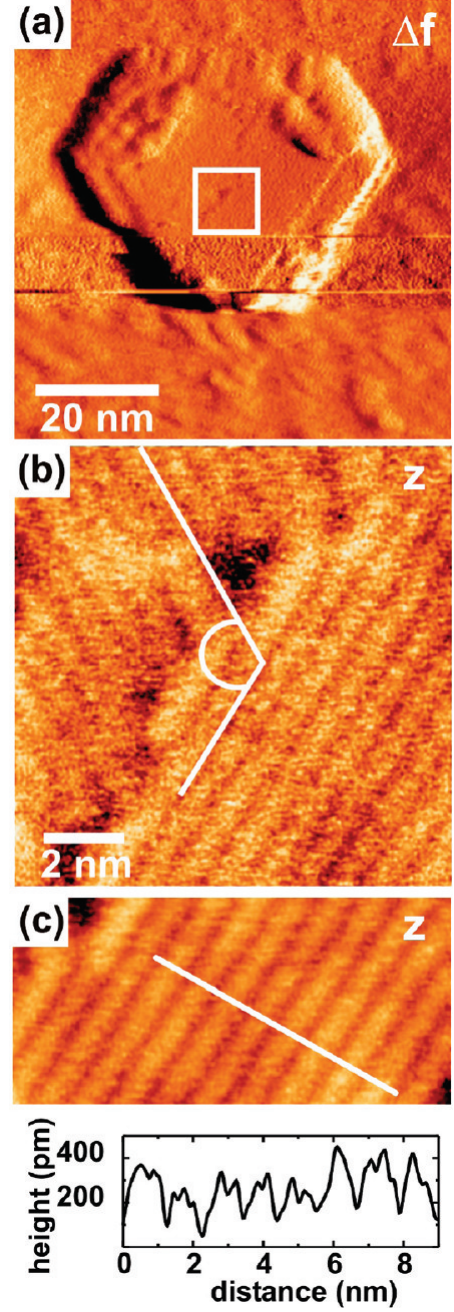

Figure 1. (a) Typical large-scale NC-AFM image of a high hexagonally shaped crystallite observed on an air-cleaved mica surface. (b) A zoom into the area marked by the square in (a) reveals a regular row structure. Rows appear in domains enclosing an angle of $117 \pm 5^{\circ}$ with respect to each other. (c) Further zooming in and reducing the tip sample distance results in a better contrast. The cross-section analysis yields equidistant rows separated by $1.02 \pm 0.05 \mathrm{~nm}$.

numbers ranging from 4500 (large frame size) to 38500 (small frame size) particles per $\mu \mathrm{m}^{2}$ were observed. ${ }^{19}$ This indicates that only a fraction of the small particles is seen in large frame size images. In our experiments we find a variety of particle sizes and shapes, including characteristic flat and regularly shaped crystallite structures as presented in Figure 1.

Figure 1a displays a hexagonally shaped crystallite structure exhibiting a fairly flat top terrace. A cross-section profile analysis of a topography mode image of the crystallite (not show here) yields a height of about $1.7 \mathrm{~nm}$ for this structure. Despite the rather high density of small particles around the crystallite (not seen in Figure 1 but in Figure $3 b$ and other images taken under similar conditions ${ }^{19}$ ), the top plane of the crystallite is free of adsorbed particles. This points to a growth of particles on the mica surface rather than a deposition after the development of the large particles. A more detailed view of the flat terraces on top the crystallite is given in the zoom in Figure 1b, revealing equidistant rows that are aligned along two preferential directions enclosing an angle of $117 \pm 5^{\circ}$. A cross-section profile analysis yields a periodicity of $b=1.02 \pm 0.05 \mathrm{~nm}$ for the mean distance of these rows (see Figure 1c). 

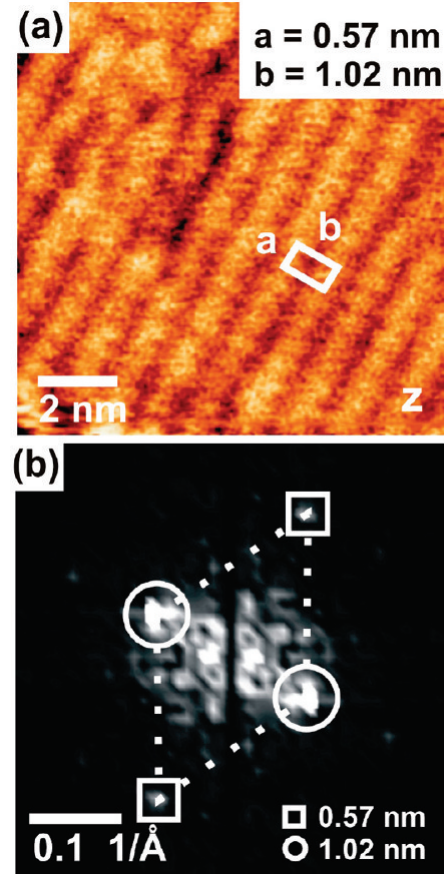

Figure 2. Highly resolved image taken on the terrace of the high hexagonally shaped crystallite introduced in Figure 1. (a) Topography image showing both the row structure presented in Figure 1c and a periodicity along the row direction. (b) Fourier transform power spectrum of image (a) revealing spots corresponding to the surface unit cell shown in (a). Spots marked by circles originate from the row periodicity of $1.02 \mathrm{~nm}$. Spots marked by squares correspond to the periodicity along the rows with $a=0.57 \pm 0.05 \mathrm{~nm}$.
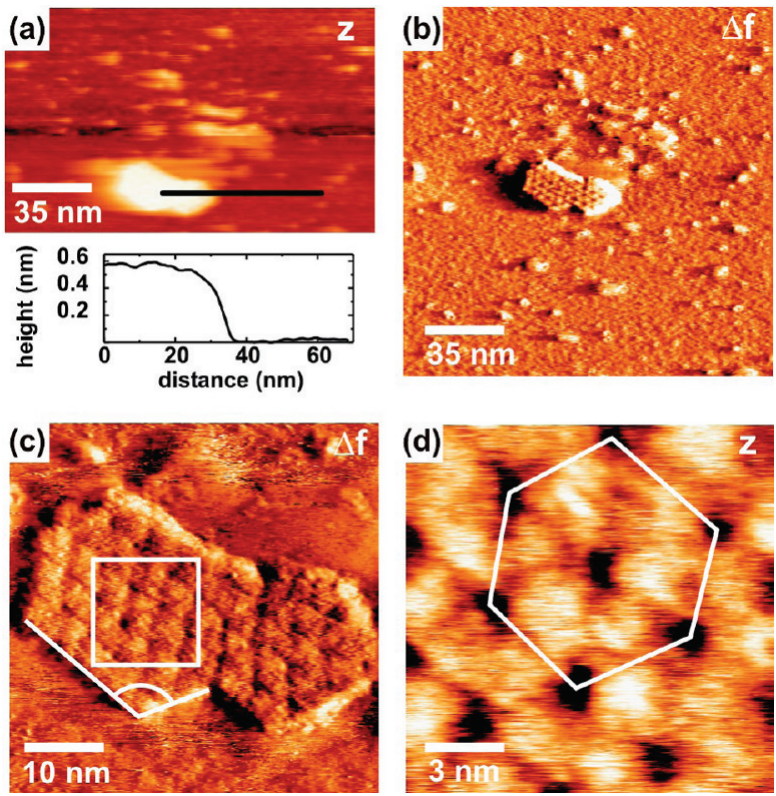

Figure 3. NC-AFM images of a flat hexagonally shaped crystallite found on the air-cleaved mica surface. (a) Large-scale topography image and cross-section analysis. (b) Constant height image of the same structure revealing the large, regularly shaped structure surrounded by smaller precipitates. (c) Zoom onto the crystallite in constant height mode exhibiting the hexagonal shape (117 \pm $5^{\circ}$ angle) and moiré surface structure. (d) Details from (c) revealing the moiré hexagon with a side length of $5.2 \pm 0.1 \mathrm{~nm}$.

By further decreasing the tip-sample distance, we can resolve more structural details on the crystallite terrace. As indicated in Figure 2a, an additional periodicity along the rows appears.
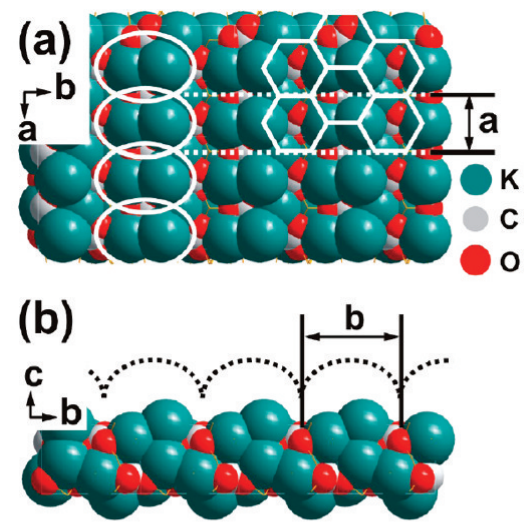

(c)
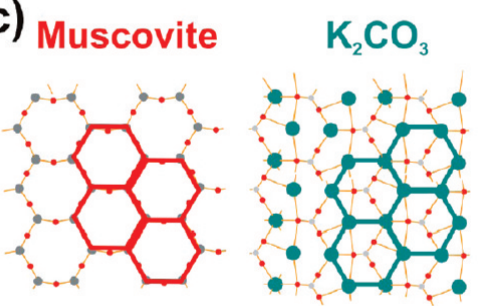

moiré structure

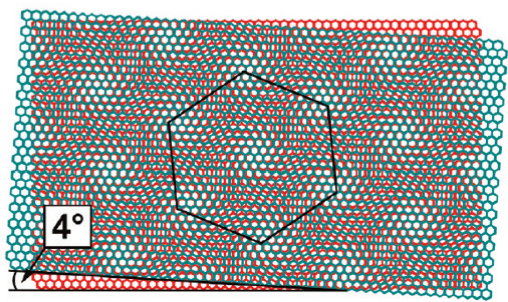

Figure 4. Structural model of a bulk-terminated potassium carbonate surface layer. Atoms are shown with their van der Waals radii. Top (a) and side (b) view of the surface structure illustrating protruding rows of neighboring potassium ions separated by $0.980 \mathrm{~nm}$. Within these rows, potassium dimers are regularly separated by a periodicity of $0.564 \mathrm{~nm}$. (c) Models highlighting the hexagonal lattices of mica (red, hexagon side length $0.52 \mathrm{~nm}$ ) and potassium carbonate (green, hexagon side length $0.564 \mathrm{~nm}$ ). The moiré pattern with a periodicity of $5.2 \mathrm{~nm}$ is obtained by the superposition of the lattices with a rotation angle of $4^{\circ}$.

To analyze the structural details of this image, a Fourier tansform power spectrum of the image from Figure $2 \mathrm{a}$ is presented in Figure 2b. The Fourier analysis yields six distinct spots, reflecting the high degree of order of the imaged surface structure. The two spots marked by circles reflect a periodicity of $1.02 \mathrm{~nm}$ perpendicular to the rows. Further spots along this direction correspond to a periodicity of $0.51 \mathrm{~nm}$ and are higher order signatures of the $1.02 \mathrm{~nm}$ periodicity. Two further spots (marked by squares) with an axis perpendicular to the axis of the other four spots reveal a periodicity of $a=0.57 \pm 0.05 \mathrm{~nm}$ along the protruding rows. This analysis establishes a surface unit cell with side lengths of $a$ and $b$, respectively, as drawn in Figure 2a.

Besides the 3-dimensional high crystallites described above, regularly shaped flat islands are observed on the surface. Images of a typical flat structure are presented in Figure 3. The crosssection analysis of the structure imaged in topography mode (Figure 3a) reveals a height of $0.6 \pm 0.1 \mathrm{~nm}$, and it is important to notice that all observed structures belonging to this second type exhibit the very same height of $0.6 \mathrm{~nm}$. The hexagonal shape of this crystallite and lateral dimensions (Figure 3b,c) are quite similar to those for the high crystallite analyzed in Figures 1 and 2. However, the high-resolution constant height image shown in 
Figure $3 b$ reveals structural details that are distinctly different from those for the high crystallites. Here we find a hexagonal pattern with a hexagon side length of $5.2 \pm 0.1 \mathrm{~nm}$, as it is most evident from the zoom image of Figure $3 \mathrm{~d}$.

The experimental evidence detailed above can answer the question whether the observed crystallite structures consist of potassium carbonate. A model of a bulk-terminated potassium carbonate crystal displaying top and side views of a layer is shown in Figure 4a. Potassium carbonate crystallizes in the hexagonal closepacked (hcp) structure, readily explaining the angle of $117 \pm 5^{\circ}$ observed between the rows in Figure $1 \mathrm{~b}$ as alternating planes in the hep structure are rotated by $120^{\circ}$. The top view of this model displays an array of $4 \times 4$ rectangular surface unit cells of the potassium carbonate structure where ions are displayed with their respective van der Waals radii. The potassium carbonate bulk unit cell ${ }^{30}$ has dimensions of $a=0.564 \mathrm{~nm}, b=0.980 \mathrm{~nm}$, and $c=0.688 \mathrm{~nm}$ and exhibits two characteristic structural features: Two neighboring potassium ions along the $b$-direction form regularly separated and protruding rows with a periodicity of $b=0.980 \mathrm{~nm}$. Within these rows, there is a periodicity of $a=0.564 \mathrm{~nm}$ along the $a$-direction describing the distance of the potassium ion dimers. This row structure and the periodicities perfectly match the observation from Figure 2 within the experimental error and provide a first piece of strong evidence that the observed high crystallites indeed are potassium carbonate nanocrystals grown with their $c$-axis perpendicular to the Muscovite surface.

At a first glance, the flat structures exhibiting a hexagonal surface pattern are difficult to interpret as the observed periodicity of $5.2 \mathrm{~nm}$ is way too large to represent any atomic lattice. However,

(30) Gatehouse, B. M.; Lloyd, D. J. J. Chem. Soc., Dalton Trans. 1973, 70. the periodicity becomes most evident when attributing it to a moiré effect, resulting from the superposition of the mica surface structure with the potassium carbonate surface structure as demonstrated in Figure 4c. A $4^{\circ}$ rotation of the two lattices precisely produces a moiré pattern with a $5.2 \mathrm{~nm}$ periodicity. Now we recall that all flat structures exhibiting the moiré pattern have the same height of $0.6 \pm 0.1 \mathrm{~nm}$, corresponding to the thickness of a single potassium carbonate sheet in the $c$-direction ( $c=0.688 \mathrm{~nm}$ ). Therefore, we interpret the flat structures as single layer potassium carbonate grown on mica where the $4^{\circ}$ angle of rotation points to an energetically favorable position of adsorption of the potassium carbonate on top of the mica surface. A single layer appears to be thin enough for observing the influence of the underlying mica lattice via the moiré pattern. We assured that the structure cannot be a moiré pattern created by the interference of a residual mica flake left behind after cleavage.

In conclusion, our results strongly support the notion of potassium carbonate growing on mica with the $c$-axis perpendicular to the surface. While the potassium carbonate surface unit cell can clearly be identified in atomically resolved images on high crystallites, potassium carbonate sheets with a thickness corresponding to the height of one bulk unit cell exhibit a nanometer size moiré pattern resulting from the superposition of the mica and potassium carbonate lattices. Both results clearly corroborate the hypothesis of potassium carbonate formation upon the interaction of a cleaved mica surface with ambient air. ${ }^{20,21}$

Acknowledgment. Financial support from the EU FP7 integrated project PicoInside and the EU Marie Curie action NEED is gratefully acknowledged. We are indebted to R. Bechstein, P. Rahe, L. Tröger, and T. Dienel for most stimulating discussions. 